\title{
EINE GEOGRAPHISCHE ÜBUNG AUF DER OBERSTUFE DER MITTELSCHULE
}

\author{
Die seit der Mitte des I9. Jahrhunderts abgegangenen Siedlungen („Wüstungen“) in der \\ Umgebung von Winterthur \\ WALTER WIRTH \\ Mit einer Kartenskizze
}

Seit dem Sommersemester 1951 bestehen in der 6. Klasse des Winterthurer Gymnasiums geographische Übungen. Über ihre Art und Durchführung sowie über die einjährigen Erfahrungen wurde in den „Geographica Helvetica“ Bd. VI, S. $44-45$ kurz berichtet. Im Sonderheft "Schweiz“ der „Geographischen Rundschau“ erschien inzwischen als Beispiel solcher Übungen eine „Siedlungsaufnahme“ (von Nußberg im Tößtal). Als 2. Beispiel möge hier eine Untersuchung über die neuern Wüstungen in der Umgebung Winterthurs folgen. Stets handelt es sich darum, die Schüler selbst an die Quellen heranzuführen. An diesem Beispiel läßt sich zudem zeigen, daß zur Lösung anthropogeographischer Probleme mitunter verschiedene Methoden aus dem Bereich des Historikers wie des Naturwissenschafters angewendet werden müssen. Natürlich sind dem Mittelschüler keine tiefschürfende Archivstudien zuzumuten. Er hat sich in diesem Gebiet vornehmlich an die kartographischen Quellen zu halten, wie sie z. B. für den Kanton Zürich in den Kartenwerken von GYGER (1667) und WILD (ca. 1850) vorliegen. Anderseits ist der Mittelschüler auch nicht zur Durchführung chemischer Bodenanalysen befähigt. Seit aber in der Molybdänblau-Methode ein einfaches Mittel besteht, Phosphatanreicherungen im Boden und damit selbst längst verlassene Siedlungsplätze nachzuweisen, sollte man nicht vor ihrer Anwendung zurückschrecken.

\section{GANG DER ÜBUNG}

Nachdem der Umfang des Untersuchungsgebietes abgesteckt war, ließ sich in gemeinsamer Arbeit durch sorgfältigen Vergleich der Wildschen Karte mit den entsprechenden Blättern der Siegfriedkarte der Bestand an neuern Wüstungen ermitteln. Durch Heranziehen der Gygerkarte ließ sich zudem ein Urteil bilden über das Alter der wüstgelegten Siedlungen. Jeder Gruppe von 2 Schülern wurde sodann eine Wüstung zur genauern Untersuchung zugewiesen. Durch ein Merkblatt sahen sie sich auf die Punkte aufmerksam gemacht, die sie im Gelände, sei es durch direkte Beobachtung, sei es durch Erkundung bei zuverlässigen, ältern Anwohnern, zu ermitteln hatten (besondere Bodenformen, Spuren der Wasserversorgung, Gebäudereste, Relikte von Kulturpflanzen - Zeitpunkt und Grund der Wüstlegung der Siedlung). Die Ergebnisse waren sorgfältig zu protokollieren und nach Möglichkeit in einen Plan 1:5000 einzutragen, den die Schüler nach der topographischen Karte zu erstellen hatten. Er sollte auch die Leitlinie mit den Entnahmestellen für die Bodenproben enthalten, die hernach im chemischen Laboratorium (Dr. Christen) der Kantonsschule zu untersuchen waren. Was das Vorgehen bei der Entnahme der Proben und deren Untersuchung nach der Molybdänblaumethode anbelangt, wurden die Schüler auf die ebenso ausführliche wie leichtfaßliche Anleitung von LоRсн, «Die siedlungsgeographische Phosphatmethode», in den «Naturwissenschaften», Bd. 1940 verwiesen ${ }^{1}$.

\section{E R G E B N I S S E}

Die im Folgenden wiedergegebenen Ergebnisse entsprechen im Wesentlichen den Protokollnotizen der Schüler. Aus ihrer Gliederung, die sich an die Punkte des Merkblattes hält, ist der Gang der Übung ersichtlich.

\section{Inventar der Wüstungen}

Im Raume Riedhof (Aesch-Neftenbach) im NW von Wallikon (Attikon-Bertschikon) im NE-Lindau im SW und Ober-Rikon im SE wurden 8 eindeutige Wüstungen registriert ${ }^{2}$ :

${ }^{1}$ Herr Prof. Frauenfelder, Technikum Winterthur, hatte mich in freundlicher Weise auf diesen Aufsatz aufmerksam gemacht.

2 Vergleiche die Siedlungskarte der Umgebung von Winterthur von H. KL.̈̈U im Atlas zur Geschichte des Kantons Zürich, 1951, S. 53, wo auch ältere, z. T. nur aus Flurnamen erschlossene wüstgelegte Siedlungen angeführt sind. 
Forrenberg, P. 565, nordöstl. Elsau (am Rand von «Alt Schmatt») ; Pfannenstiel, P. 502, nordwestl. Elsau (beim «Schönholz») ; Etzberg, P. 567, innerhalb des «Eichbühl», nordöstl. Seen; Schafenberg, P. 551 bei Thalacher nördl. Ricketwil; Tugstein, südwestl. Seenerrüti (Sennhof-Kollbrunn); Linsenthal, P. 472, nördl. Kyburg; Klausacker, P. 632 westl. Billikon (Ottikon); Schnabelacker, östl. Ettenhausen (Kyburg).

Alle wüstgelegten Siedlungen - mit Ausnahme von «Etzberg》 und «Linsental », die schon um 1667 existierten - müssen jüngere Gründungen sein.

II. Untersuchung einer Wüstung am Beispiel von Tugstein (mit gelegentlichen Hinweisen auf «Pfannenstiel » und «Etzberg»).

1. Lage von Siedlung und Flur nach dem topographischen Atlas der Schweiz (B1. 68, Turbenthal) [s. Kartenskizze].

2. Festlegung des Siedlungsplatzes und der Nutzung der Flur nach Beobachtungen im Gelände.

a) Nach der Bodenform: Der im übrigen leicht geböschte Hang zeigt gegen den obern Waldrand hin, bei P. 540 eine deutliche Verebnung, die sich auch durch ihre mehr oder weniger rechteckige Abgrenzung als früherer Siedlungsplatz zu erkennen gibt.

b) Nach Spuren der Wasserversorgung: Am obern Waldrand entspringt eine Quelle. Noch deutet ein eisernes Rohr darauf hin, daß sie für die Siedlung gefaßt war. Heute rinnt das Wasser in einem Gräbchen gegen W einem Tobel zu und kann zu Zeiten der Wiesenwässerung dienen.

c) Durch Gebäudereste: Der ehemalige Wohnplatz fällt durch die relativ magere Vegetation auf. Bei der Entnahme von Bodenproben, die das Abheben der obersten Schicht erforderte, kamen Bauschutt, kohlige Reste und Trümmer von (Flach-)Ziegeln zum Vorschein.

d) Durch Reste von Kulturpflanzen: Noch steht in nächster Nähe des ehemaligen Siedlungsplatzes ein verwilderter Obstbaum. Mehrere solcher Bäume sollen

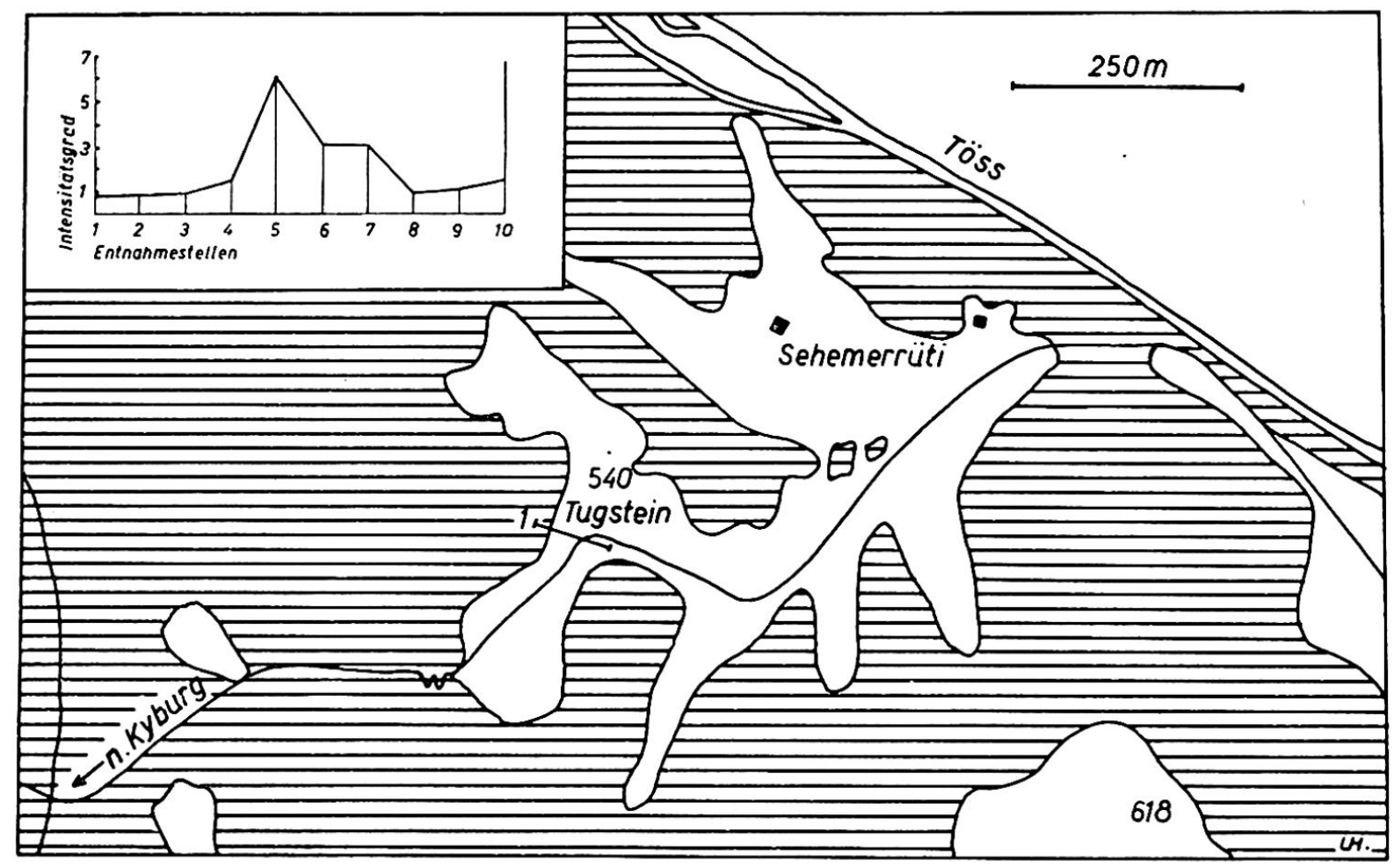

Das Gebiet von Tugstein. Nach Bl. 11, Kyburg, der Karte des Kantons Zürich 1:25 000, Zürich 1852 bis 1865 (Wildsche Karte). Links oben: Phosphatkurve Profil 1 im Kärtchen. 
nach der Aussage des Besitzers des Nachbarhofes «Seenerrüti » in den letzten Jahrzehnten umgehauen worden sein. Als Zeugen des frühern Gartens kommen jedes Frühjahr Narzissen zum Aufblühen. (Der «Garten» der Wüstung 《Pfannenstiel » ist der hohen Futtererträge wegen geschätzt.)

e) Durch Spuren früherer Bebauung: Die Flur des «Tugstein 》 läßt keine solche erkennen. Wohl aber treffen wir inmitten des Waldbestandes, der die Flur der ehemaligen Siedlung «Etzberg » einnimmt, und zwar besonders südlich der «Arisstraße » übereinander mehrere, durch kurze Steilborde getrennte, schmale Terrassen, die der früheren Beackerung zu verdanken sind.

f) Durch Phosphatanreicherung im Boden: Längs einer ostwestlich verlaufenden Leitlinie, die von Waldrand zu Waldrand und durch den vermuteten Siedlungsplatz geht, wurden im Abstand von je $10 \mathrm{~m}$ Bodenproben entnommen und diese im Laboratorium nach der Molybdänblaumethode untersucht. Die «Phosphatkurve» hat auf dieser Strecke den im Kärtchen links oben angegebenen Verlauf, und weist mit der Anschwellung bei Punkt 5 auf die ehemalige Siedlungsstelle hin ${ }^{3}$.

\section{Zeitpunkt auf Grund der Wüstlegung}

Der Hof «Tugstein » ist nach der Aussage des Besitzers der «Seenerrütti 1870 abgebrannt und im Gefolge nicht wieder aufgebaut worden. (Dasselbe Schicksal erreichte 1860 den Hof «Pfannenstiel ». Hier ist näher bei der Hauptsiedlung Elsau der Hof «Einfang» entstanden, von dem aus heute die Grundstücke der Wüstung größtenteils bewirtschaftet werden. Dagegen verschwand beim «Etzberg» mit dem Hof auch die Flur. Die ehemalige Waldlichtung wurde mit Nadelhölzern aufgeforstet).

${ }^{3}$ Bei Beigabe eines „Entwicklers“ zur Bodenprobe im Reagenzglas und Erhitzen im Wasserbad tritt je nach dem Phosphatgehalt der Probe eine Blaufärbung von verschiedener Intensität ein. Für ca. 30 Proben verwendet man einen Entwickler, bei dem $6 \mathrm{~g}$ Ammoniummolybdat, $5 \mathrm{~g}$ Natriumsulfit, $1 / 4 \mathrm{~g}$ Hydrochinon in $550 \mathrm{~cm}^{3}$ destilliertem Wasser $+45 \mathrm{~cm}^{3}$ Schwefelsäure gelöst sind. - Die Intensität der Blaufärbung der Bodenproben kann durch Vergleich mit Standardlösungen (von Entwickler + Dinatriumphosphat in verschiedener Verdünnung) leicht geschätzt werden.

\section{GEBUR'TSTAGE}

\section{LE PROFESSEUR CHARLES BIERMANN A 80 ANS}

Le 9 mars de cette année, le professeur ChARLES BIERMANN a feté son quatre-vingtième anniversaire. "Geographica Helvetica" tient à féliciter son fidèle collaborateur et joint de tout cœur son hommage à ceux qui sont déjà montés vers la paisible demeure du Mont.

Né à Lausanne le 9 mars 1875 , Charles Biermann y fit toutes ses études. Le jeune licencié songeait à faire carrière d'helléniste quand il fut appelé au Collège classique cantonal en 1897. Mais l'homme propose... et MAURICE LUGEON, qui enseignait alors la géographie physique à Lausanne, l'entraina dans son sillage pour en faire un géographe. Docteur ès lettres en 1907 avec la thèse * La Vallée de Conches, en Valais ", il fut chargé d'un cours de géographie économique en 1912 et devint titulaire de la chaire de géographie à l'Université de Lausanne en 1926. Partagé entre son enseignement au Collège et des cours à l'Université, BIERMAnN se soumet à un rythme de travail qu'il n'abandonnera jamais et qui fait l'admiration de ses collègues. Bien plus, dès 1921 , il consacre tous ses mercredis à l'Université de Neuchâtel où il succède à Charles KNapp. La même année, il se voit confier la direction du «Bulletin de la société neuchâteloise de géographie», publication qu'il dirigea brillamment pendant un quart de siècle. Les charges dont il était revêtu désignaient tout naturellement le professeur BIERMANn à remanier l'édition des manuels-atlas de Rosier, en usage dans toutes les écoles de la Suisse romande. Il fera aussi partie de la commission chargée de l'édition de l'Atlas scolaire suisse. En 1934, il préside la Fédération des sociétés suisses de géographie et la Société neuchâteloise le délègue à de nombreux congrès internationaux où il prend une part active.

Toutes ces activités n'empêchent pas ChaRLES BIERMANN de présenter une cinquantaine de travaux qui le montrent curieux de tous les domaines de la géographie. C'est pourtant au canton de Vaud qu'il devait réserver le meilleur de lui-même, à ce canton qui attendait encore son interprète. Déjà en 1910, une étude du professeur lausannois sur le " Jorat" indiquait où ses recherches allaient de préférence se porter. Puis ce furent les longues enquêtes qui devaient aboutir en 1947 à la publi- 